\title{
Enhancing hybrid metal- semiconductor systems beyond SERS with PIERS (photo-induced enhanced Raman scattering) for trace analyte detection
}

Glass, D., Cortés, E., Peveler, W., Howle, C., QuesadaCabrera, R., et al.

D. Glass, E. Cortés, W. J. Peveler, C. R. Howle, R. Quesada-Cabrera, I. P. Parkin, S. A. Maier, "Enhancing hybrid metal-semiconductor systems beyond SERS with PIERS (photo-induced enhanced Raman scattering) for trace analyte detection," Proc. SPIE 11416, Chemical, Biological, Radiological, Nuclear, and Explosives (CBRNE) Sensing XXI, 1141602 (24 April 2020); doi: 10.1117/12.2557517

SPIE. Event: SPIE Defense + Commercial Sensing, 2020, Online Only 


\title{
Enhancing hybrid metal-semiconductor systems beyond SERS with PIERS (Photo-induced enhanced Raman scattering) for trace analyte detection
}

\author{
D. Glass*a, b, E Cortés ${ }^{\mathrm{a}, \mathrm{c}}$, W. J. Peveler ${ }^{\mathrm{d}}$, C. R. Howle ${ }^{\mathrm{e}}$, R. Quesada-Cabrera ${ }^{\mathrm{b}}$, I. P. Parkin ${ }^{\mathrm{b}}$, S. A. \\ Maier*a, c

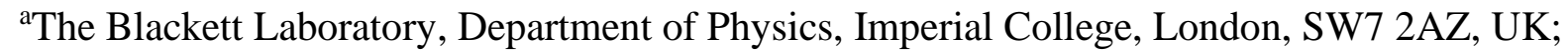 \\ ${ }^{b}$ Department of Chemistry, University College London, 20 Gordon St., London, WC1H 0AJ, UK; \\ ${ }^{c}$ Chair in Hybrid Nanosystems, Nanoinstitute Munich, Ludwig-Maximilians-Universität München, \\ 80799, München, Germany; \\ ${ }^{\mathrm{d} S c h o o l ~ o f ~ C h e m i s t r y, ~ J o s e p h ~ B l a c k ~ B u i l d i n g, ~ U n i v e r s i t y ~ o f ~ G l a s g o w, ~ G l a s g o w, ~ G 12 ~ 8 Q Q, ~ U K ; ~}$ \\ ${ }^{\mathrm{e}}$ Defence Science and Technology Laboratory, Porton Down, Salisbury, Wiltshire, SP4 0JQ \\ *stefan.maier@physik.uni-muenchen.de; www.hybridplasmonics.org/
}

\begin{abstract}
Hybrid metal-semiconductor systems are promising substrates for field Raman analysis due to their ability to use both electromagnetic and chemical enhancement pathways for surface enhanced Raman spectroscopy (SERS). Photo-induced Raman spectroscopy (PIERS) has previously been shown to be a promising method utilizing an additional enhancement route through photo-inducing atomic surface oxygen vacancies in photocatalytic metal-oxide semiconductors. The photoinduced vacancies can form vibronic coupling resonances, known as charge transfers, with analyte molecules, enhancing the signal beyond conventional SERS enhancements. However, conventional UV sources most often used for excitation of the PIERS substrate are impractical in combination with portable Raman systems for field analysis. In this work we show how a small UVC LED, centered at $255 \mathrm{~nm}$, can replicate the same results previously reported with the benefit of allowing greater in-situ real time measurements under constant UV exposure. The UV LED source can be controlled more easily and safely, making it a practical UV source for field PIERS analysis.
\end{abstract}

Keywords: SERS (surface enhanced Raman spectroscopy), PIERS, oxygen vacancy defects, metal oxide, chemical sensing, enhanced detection

\section{INTRODUCTION}

Raman spectroscopy is common technique employed for molecular characterization and identification. ${ }^{1-4}$ Through inelastic vibrational interactions, Raman spectroscopy relies on specific vibrational modes in a molecule. As each Raman band can be assigned to specific molecular vibrational modes, a Raman spectrum can be de decoded to allow molecular "fingerprint"-like detection. Substrates used for detection applications are often tailored to specific functional groups or molecule "families", whereas the use of Raman spectroscopy presents a non-chemical specific method for analyte detection. Raman spectroscopy itself can be considered a relatively weak spectroscopy due to low molecular cross sectional areas, in comparison to infrared spectroscopy for example. The discovery of SERS (surface enhanced Raman spectroscopy) overcame this problem and helped to establish Raman spectroscopy as the popular and powerful analytical technique it is today. ${ }^{5}$ SERS was first discovered on roughened silver surfaces, but modern systems often use a wide range of noble- 
metal based substrates. ${ }^{6-8}$ With the advancement of substrate manufacturing techniques, the resultant Raman enhancement factors have greatly advanced over time, enabling modern SERS substrates to detect single molecules. . $^{1,7,8}$

The exact mechanism behind the measured enhancements is debated within the literature, where many different models have been presented to explain this phenomena over the years. ${ }^{6,7,9,10}$ Roughened surfaces can locally focus and intensify electromagnetic (EM) fields through local surface plasmonic effects, the collective movement and oscillation of valence electrons. 5 This results in EM fields being highly focused to nanoscale volumes, known as "hot-spot" regions. Hot spot regions can both enhance the effective local incident field on the Raman analyte enhance the re-emitted Raman scattering. ${ }^{11}$ The resultant Raman band enhancements are often presented as enhancement factors (EF), ${ }^{5}$ typically reported to be around $10^{6}$ for noble-metal substrates although theoretically they can exhibit much higher values. ${ }^{7}$ The use of nanostructured noble metals has often been referred to as "conventional SERS". For these materials the EM enhancement pathway is often responsible for the majority of the SERS enhancement and other enhancement mechanisms play a less significant role.

However, the concept of an EM enhancement pathway cannot be used alone to explain the source of all Raman enhancements. For example, semiconductors are commonly used alternative SERS substrates over the last few decades. 6,12-16 Enhancements of these substrates primarily relies on a chemical enhancement (CE) mechanism. Often the EF for these materials is found to be significantly lower than noble metal enhancements, usually between 1-100. However, recent reports using defective metal oxides have shown comparable CE to EM enhancements. ${ }^{17,18}$ This mechanism primarily relies on interactions and changes between charge distributions and vibronic states, resulting in vibronic coupling resonances between the analyte and the surface. ${ }^{13,14,19}$ More recent works have attempted to utilize both enhancement mechanisms in a synergistic SERS approach using a metal-semiconductor hybrid system.

An alternative and novel method for further increasing the SERS intensities utilizes defective semiconductor materials. Inducing surface defect states into metal-oxide semiconductors (MOS) can largely change a variety of physical and chemical properties of the material. In addition, surface defect states can interact with adsorbed molecules, resulting in an additional CE pathway. ${ }^{20-22}$ Surface oxygen vacancies, $\mathrm{V}_{\mathrm{o}}$, induced through high energy UV irradiation (254 nm) on photocatalytic MOS substrates has come to be known as PIERS (Photo-Induced Enhanced Raman Spectroscopy). The mechanism is thought to rely on photo-induced carrier production, where induced carrier lifetimes are increased due to carrier separation due to the hybrid metal-semiconductor system. Holes induced near the surface of the MOS can weaken surface oxygen bonds and/or mediate splitting reactions with surface adsorbed $\mathrm{H}_{2} \mathrm{O}$ and $\mathrm{O}_{2}$. As a result the concentration of surface oxygen vacancies is increased and an increase in Raman band enhancements is seen. Under ambient conditions, or exposure to $\mathrm{O}_{2}$ or $\mathrm{H}_{2} \mathrm{O}$, the induced vacancies were shown to "heal", decreasing the Raman band intensities back to the original background enhancement level. ${ }^{22}$

Previous PIERS studies conducted have primarily used fixed laboratory UV sources, typically UV gas discharge lamps, which are generally larger, have significant power supply requirements and cannot be as easily adjusted and are therefore hard to incorporate into portable Raman systems for field analysis. In addition, due to the size and nature of the UV lamps, real time Raman measurements under UV exposure are challenging practically. Although enhancements under constant UV exposure have been reported previously, ${ }^{22}$ the UV source was reported to be switched off during measurements where objects often had to be refocused on the substrate before taking measurements between UV exposures, due to experimental constraints - see Figure 1. In this paper we report an alternative UV source which can overcome all these issues, allowing real time Raman monitoring under UV irradiation, a UV LED. A UV LED, with a peak wavelength centered at $255 \mathrm{~nm}$, allows easy illumination of the sample whilst still allowing microscope objectives to be in focus on the sample for Raman analysis, due to its small size, as shown in Figure 1b. Using the LED source the dynamics for induced vacancy formation and healing can be determined, as previously shown with a UV lamp. In addition, UV LEDs can easily be incorporated into a portable Raman system for field analysis, where the source can be safely focused onto the sample without exposing the user to UV irradiation. 
a)

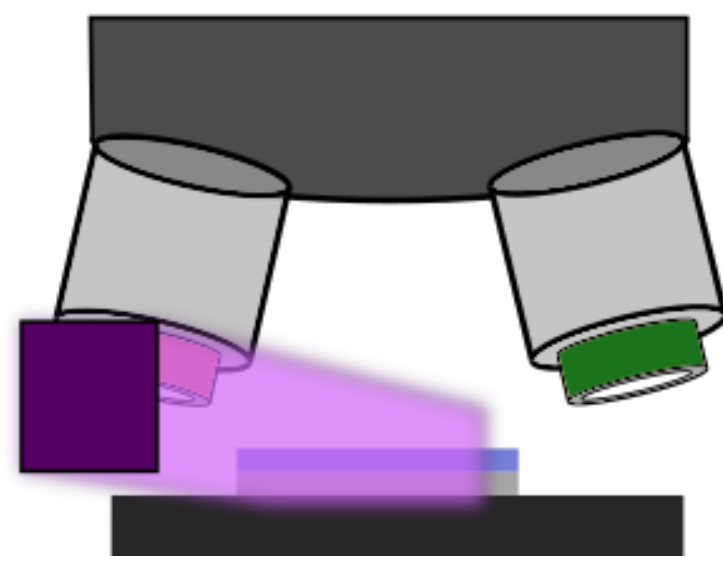

b)

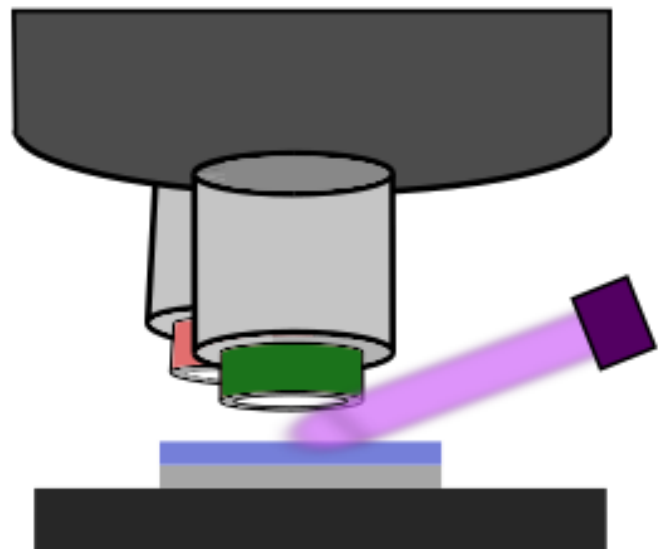

Figure 1. (a) UV Lamp illuminating substrate directly from the front, as reported previously, where objective is moved to the side to allow exposure of the substrate (previous study). (b) UV pen/LED illuminating substrate from the side with objective in place above the substrate, thereby allowing real time measurements under UV (this study).

\section{METHODOLOGY}

\subsection{Substrate synthesis}

$\mathrm{TiO}_{2}$ thin films were synthesized using aerosol-assisted vapour deposition (AA-CVD), on to quartz substrates. The substrates were washed in acetone and placed into the CVD reactor which consisted of a $320 \mathrm{~mm}$-long graphite heating block accommodated within a quartz tube with three Whatman heater cartridges. Titanium (IV) isopropoxide (Sigma Aldrich, $97 \%, 0.5 \mathrm{ml}$ ) in methanol (Fisher Scientific HPLC grade, $20 \mathrm{ml}$ ) was used as precursor and solvent respectively. An ultrasonic humidifier (Liquifog, Johnson Matthey operating at $2 \mathrm{MHz}$ ) was used to form aerosol droplets of a precursor solution which were transported to the heated rector chamber under the flow of nitrogen gas $(B O C)$ at a flow rate of $1 \mathrm{~L}$ $\mathrm{min}^{-1}$. $\mathrm{TiO}_{2}$ films were initially deposited at $500{ }^{\circ} \mathrm{C}$ and subsequently annealed to $1000{ }^{\circ} \mathrm{C}$ in air for 10 hours and then allowed to cool to room temperature to obtain a pure Rutile phase. This was confirmed by X-ray diffraction and Raman spectroscopy, where no traces of anatase were detected.

\subsection{Chemical and substrate preparation}

4-mercaptobenzoic acid (MBA, Sigma Aldrich) was dissolved in ethanol (VWR Chemicals) to create a $1 \times 10^{-5} \mathrm{M}$ solution. $0.1 \mathrm{ml}$ of the MBA solution was dropped into $0.5 \mathrm{ml}$ of $40 \mathrm{~nm}$ citrate capped AuNP solution, purchased from SPI supplies. The solution was left overnight to allow MBA to functionalize the AuNPs by replacing the citrate capping layer. The solution was then centrifuged using an Eppendorf Minispin at 6 kRPM for 6 minutes to cause the Au NPs to pellet. As much of the remaining solution as possible was removed, leaving the NPs, and deionized water was added to make a total volume of $1 \mathrm{ml}$, re-dispersing the functionalized NPs. This process was repeated 5 times to remove any unbound $\mathrm{MBA} /$ citrate from the solution. After the final washing, enough DI water was added to have $0.5 \mathrm{ml}$ of MBA functionalized $\mathrm{Au}$ NP solution. $1 \mu \mathrm{L}$ of MBA functionalized AuNP solution was dropped onto the prepared $\mathrm{TiO}_{2}$ films. The films were then placed on a hotplate which was heated with the substrate on it. Temperatures were limited to $100^{\circ} \mathrm{C}$, however samples were removed as soon as the droplet had evaporated, normally occurring after 15-30 seconds, before the hotplate had reached maximum temperature. A ring of AuNP of roughly $2 \mathrm{~mm}$ in diameter was visible on the substrates.

\subsection{UV irradiation procedures}

Two types of irradiation procedures were used, firstly an initial irradiation period and subsequent Raman measurements and secondly Raman measurements under continual constant UV exposure. Where samples were first exposed to an initial irradiation period, substrates were then placed under either UV source for 45 minutes prior to Raman analysis. For the UV lamp (UVItec LI $215 \mathrm{G}$ model $(\lambda=254 \mathrm{~nm})$ ), substrates were placed a distance of $1 \mathrm{~cm}$ away from the bulbs. For the UV 
LED (Crystal IS OPTAN-255P-SMD ( $\lambda$ centered at $255 \mathrm{~nm}$ )), the LED was powered using a bench power supply (Tenma 72-2535) at $8 \mathrm{~V}$ focused using $2 \mathrm{UV}$ grade fused silica lenses (ThorLabs LA4647-UV), collimating and subsequently focusing the LED through a lens tube. The LED set-up was placed at a distance of the focal length away from the sample, which was roughly $2 \mathrm{~cm}$. At the focal length the LED illuminates a $2 \mathrm{~mm}^{2}$ area.

For Raman measurements under continuous UV exposure, only the UV LED was used in this study. The LED was positioned to irradiate the substrate from the side at a distance where the LED was focused unto the substrate. The LED was turned on after an initial Raman measurement, using the bench power supply at $8 \mathrm{~V}$, and was kept on subsequently until all measurements were taken.

\subsection{Characterization techniques}

Raman studies were conducted using a confocal Raman microscope (WiTec) equipped with a He-Ne laser $(\lambda=633 \mathrm{~nm})$ at an average power of $1 \mathrm{~mW}$. For the measurements after an initial UV exposure a bright field objective (Zeiss 100x NA 0.8 ) was used, whereas for measurements under continuous UV LED exposure a different bright field objective was used (Zeiss 20x NA 0.4). The focal distance of the 100x and 20x objectives is roughly $1 \mu \mathrm{m}$ and $3 \mathrm{~mm}$ from the surface of the films, respectively. Hence in order to exposure the sample to UV LED irradiation whilst still taking Raman measurements the 20x objective was used as the 100x objective blocks exposure to the sample. The acquisition time for individual spectra was set at 3 seconds, where an average of 3 consecutive spectra were taken to produce the resultant spectra. Where individual spectra were taken, both after an initial UV exposure and under constant UV exposure, a series of spectra at the same position were taken over time every 5 minutes. For these measurements the measured positions were only exposed to the He-Ne laser during data acquisition. The measurements reported here represent an average of 15-20 positions taken across multiple samples. For continuous Raman measurements at a single position the acquisition time was set as above with 10 second intervals between measurements. The He-Ne laser was continuously exposed to the measured position for this type of measurement due to its nature.

\section{RESULTS AND DISCUSSION}

Raman analysis of $\mathrm{TiO}_{2}$ films showed characteristic Raman bands corresponding to the rutile phase at $438 \mathrm{~cm}^{-1}$ and 602 $\mathrm{cm}^{-1}$ respectively, as confirmed with other published spectra for $\mathrm{TiO}_{2}$. After depositing 4-mercaptobenzoic acid (MBA) functionalized onto AuNPs on the surface of the $\mathrm{TiO}_{2}$ films additional bands centered around $1065 \mathrm{~cm}^{-1}$ and $1575 \mathrm{~cm}^{-1}$ were visible, attributed to $v 8$ and $v 12$ aromatic ring vibrations of MBA, respectively. ${ }^{23}$ Under UV irradiation, on occasion, additional Raman bands were visible. These bands only appeared when the UV source was on, therefore it was assumed these bands corresponded to vibrational modes excited from the UV source rather than the He-Ne source. These bands were found to not overlap the spectral regions where $\mathrm{TiO}_{2}$ or $\mathrm{MBA}$ bands were present, therefore for the sake of this study they were discounted. UV irradiation of a sample under ambient conditions results in three time-dependent processes which affect the measured Raman band intensity concurrently occurring; laser induced photo-bleaching, vacancy formation and vacancy healing. These three processes were reported to be independent of one another, and therefore could be considered separately. A 3 step-analysis approach was followed to analyze series of Raman spectra without UV, after UV and under constant UV, following the procedure and analysis reported previously. ${ }^{22}$ Firstly, the rate of decay of the Raman band intensities due to photo-bleaching was measured for each different experimental set-up under identical conditions, excluding UV exposure. The second step analyzes samples after an initial exposure to UV irradiation, allowing the rate of induced vacancy healing to be determined. Finally, measurements were taken under constant UV exposure allow the rate of induced vacancy formation to be calculated. To investigate the UV LED source, the induced vacancy healing dynamics were first compared to that of the UV lamp.

\subsection{UV source comparison - induced vacancy healing dynamics}

Prepared substrates were exposure to UV light from either the UV lamp or UV LED for 45 minutes. The relative decay of Raman bands corresponding to MBA were monitored over time, where an average decay of the two main bands at 1065 $\mathrm{cm}^{-1}$ and $1575 \mathrm{~cm}^{-1}$ was taken. 8 random positions were analyzed per sample, where the experiment was repeated on 
multiple samples. An average of the relative decay across all positions and all samples for each UV source (PIERS decays) and for no UV exposure (SERS decay) were taken to determine the representative average decay, respectively. A relative change of signal enhancement was taken, as oppose to absolute changes in measured Raman counts, to account for changes in SERS enhancements due to hot-spot effects. By measuring the relative change in enhancement, the changes can directly correspond to photo-bleaching and/or vacancy induced affects respectively.

Figure 2a shows the average relative change in measured Raman band intensities over time, relative to the decay due to photo-bleaching alone - the blue-dotted decay. It is presented this way as after a long period of time, where all induced vacancies have healed, it can be assumed the PIERS decays would decay down to the same level as the SERS decay. It can clearly be seen that an additional enhancement of Raman bands were found after UV exposure from both sources. It is important to note that taking relative changes in enhancements can lead to non-realistic enhancements of the data. To ensure the enhanced decay is due to induced vacancy effects, Figure $2 b$ shows the changes in average Raman band intensities relative to the initially measured positon. If no vacancy effects were present the rate of decay here would be comparable to the SERS decay, which is clearly not the case. Hence we can assume induced vacancy effects were present after exposure to both the UV lamp and UV LED. The average relative enhancement factor (EF), relative to the SERS enhancement, for the lamp was found to higher than for the LED, as shown in Figure 2a. In addition, a slight change in measured behavior between the UV LED and the UV lamp can be seen in Figure 2a. These differences may be explained by the significant difference in irradiance power, Table 1.
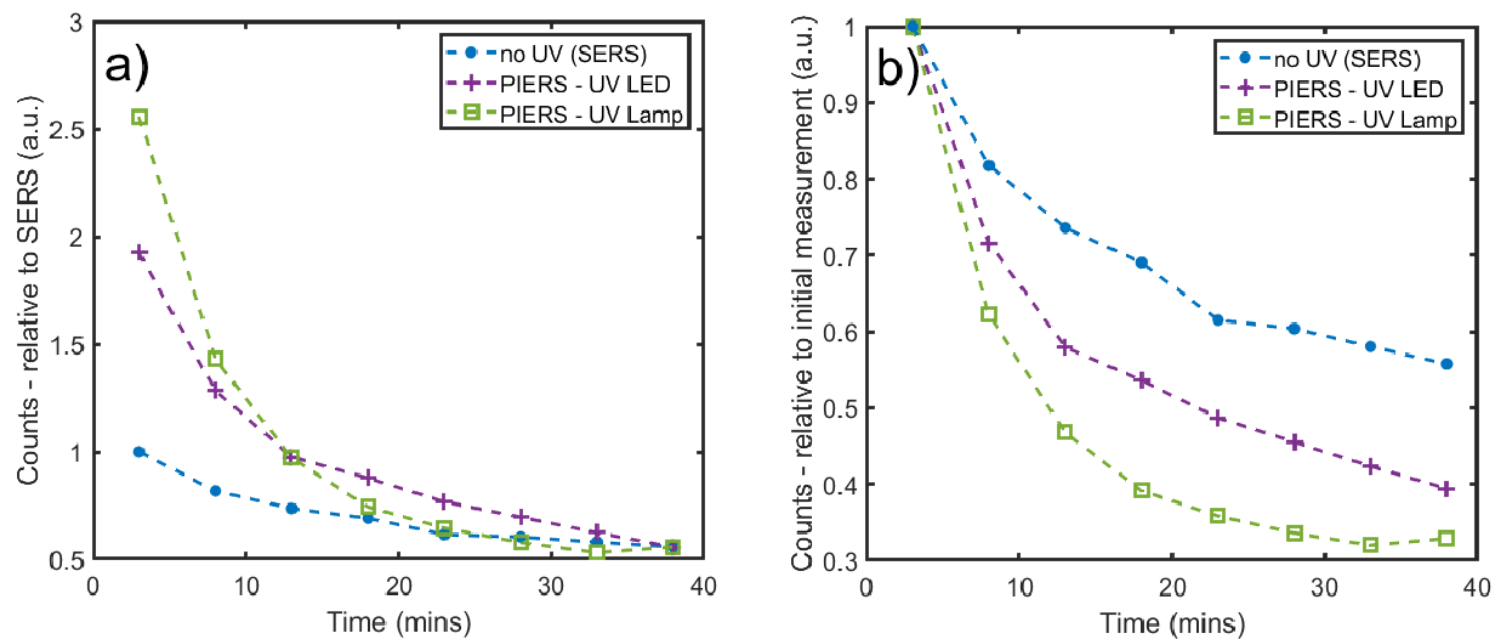

Figure 2. Average decay of Raman bands (a) relative to SERS photo-bleaching and (b) relative to the initial measurement over time after exposure to different UV sources; no source (blue, ๑), UV LED (purple, + ) and UV lamp (green, $\boldsymbol{\square}$ ). A noticeable difference between the decays after exposure to the different sources and with no source can be seen.

After the presence of an enhanced decay, and therefore induced vacancies were confirmed, the relative change in induced vacancy concentration can be calculated, Figure 3 . The respective lifetimes associated are reported in Table 1. Within error the calculated times were found to be comparable. The determined lifetime for LED induced decays had a noticeably higher error associated with it than that calculated for the lamp. The primary reason for this relies on the chosen fitting approximations, based on the model presented previously. ${ }^{22}$ The model assumes the healing of induced vacancies can effectively be modelled as an exponential decay. Although this is shown to be accurate for the UV lamp data, after 15 minutes the LED data appears to behave more like a linear decrease rather than an exponential decay. This results in an increased error in the fitting parameters, resulting in an overall increased error in the calculated lifetime. 
Table 1. Comparison of UV sources.

\begin{tabular}{|c|c|c|c|}
\hline UV Source & $\begin{array}{c}\text { Maximum power of source } \\
\text { measured }\left(\mathbf{m W} / \mathbf{c m}^{\mathbf{2}}\right)\end{array}$ & $\begin{array}{c}\text { Average EF } \\
\text { (relative to SERS) }\end{array}$ & $\begin{array}{c}\text { Calculated Vo healing } \\
\text { lifetime (mins) }\end{array}$ \\
\hline No source & 0 & 1 & N/A \\
\hline Lamp & 14 & 2.56 & $5.51 \pm 0.44$ \\
\hline LED & $1.3($ at $8 \mathrm{~V})$ & 1.92 & $7.41 \pm 2.41$ \\
\hline
\end{tabular}

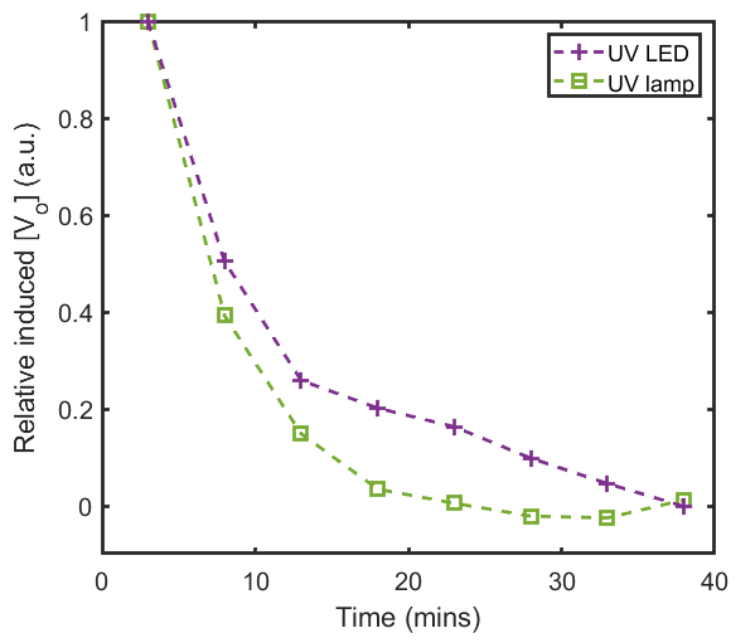

Figure 3. Calculated relative concentration of UV induced vacancies over time upon exposure to air for UV LED (purple, +) and UV lamp (green, $\square$ ).

\subsection{Constant UV LED exposure}

After measuring and calculating the rate of photo-bleaching and the rate of induced vacancy healing, dynamics for the formation of UV induced oxygen vacancies can be determined. A series of Raman spectra were taken under constant UV exposure at both single positions over time and an average of multiple positions across the surface, as shown in Figure 4. There were two main differences between these scenarios. Firstly, for single positions the objective was fixed at a constant position, hence the Raman laser was constantly exposed to the measured position. In contrast, where multiple positions were measured the Raman laser was only exposed to each position at the time data was acquired. This difference primarily affects the amount of photo-bleaching that can occur during the experiment. Secondly, a single position shows real time changes over the measured position, whereas an average of multiple positions represents the average change over the whole sample. It is important to note that even though a single position was analyzed, this measurement was repeated multiple times and the displayed data represents an average of these repeat measurements.

Figure 4a shows the changes in relative Raman counts over time for a single position on the surface with and without UV LED irradiation. An almost identical trend was seen over time, regardless of UV irradiation or not. The data above has shown that the UV LED is able to induce vacancies; however, no significant increase in Raman enhancement is seen here. In contrast, Figure $4 \mathrm{~b}$ shows a noticeable difference between the average measured Raman enhancement across the surface with and without constant UV LED irradiation. Although a clear overall decay can be seen with and without UV irradiation, a slower decay rate can be seen with the UV LED on. It is interesting to note after 5 minutes of UV exposure the measured Raman intensity was found to be higher than the initial average intensity. This observation can be explained by the rate of vacancy formation dominating over the rate of photo-bleaching during this time. Vacancy healing effects are considered significantly reduced during this time window, as it is within the healing lifetime of the first induced vacancies. At later times effects due to combined vacancy healing and laser induced photo-bleaching dominate over enhancements due to additional induced vacancies, therefore an overall decrease in enhancement is seen. From the comparison between measurements made at a single position, Figure $4 \mathrm{a}$, and the representative average across the surface, Figure $4 \mathrm{~b}$, it is 
reasonable to assume that little-to-no significant clusters of vacancies were induced at the single position measured. Additionally, any vacancies which were induced at these positions were obscured by photo-bleaching affects.
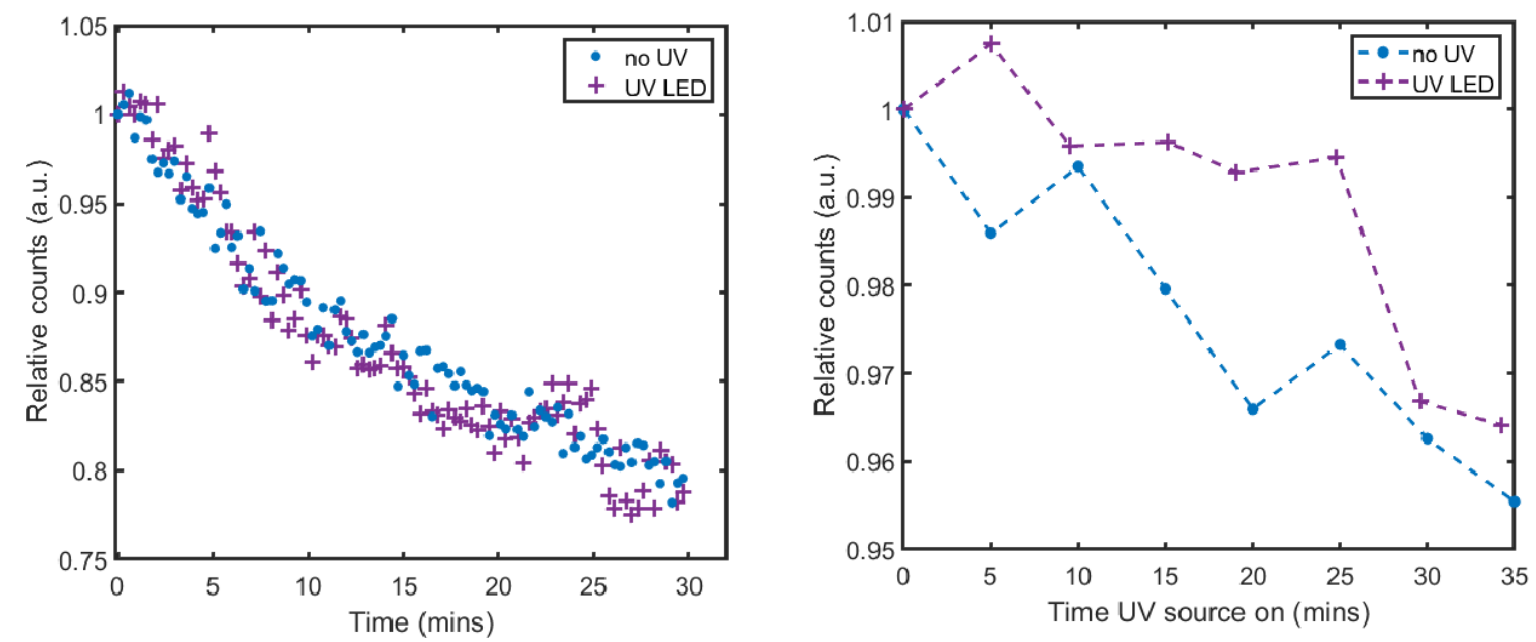

Figure 4. Raman counts relative to the initial Raman band enhancement for: (a) an average of single positions measured over time without UV (blue, $\bullet$ )) or with constant UV LED exposure (purple, +), (b) an average of 10-20 positions across the surface of the film over time without UV(blue, $\bullet$ ) or with constant UV LED exposure (purple, +).

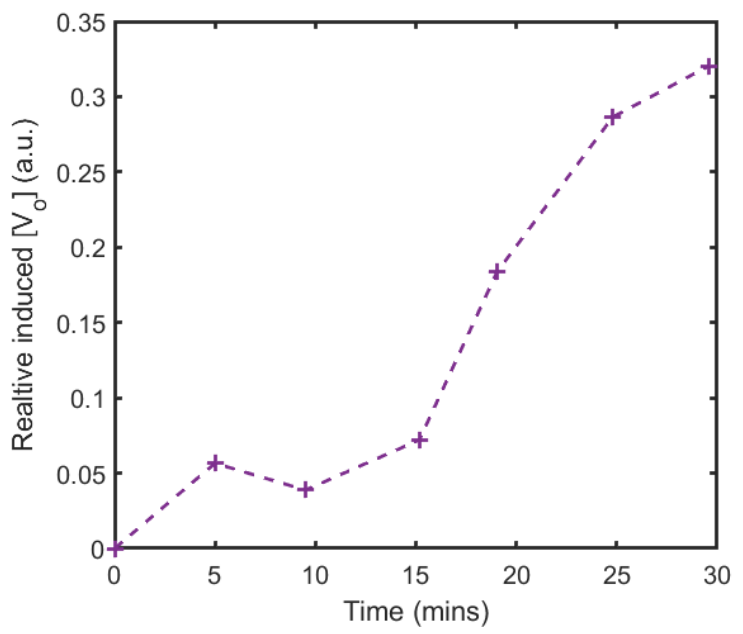

Figure 5. Calculated average relative amount of UV LED induced vacancies over time.

Figure 5 shows the calculated formation of induced vacancies over time derived from the data shown in Figure 4b. The relative concentration of induced vacancies is 0 at 0 minutes as this measurement was conducted prior to switching on the UV source. Subsequent times correspond to the time exposed to UV irradiation. This data was calculated following the procedure reported previously, after taking account effects of both photo-bleaching and vacancy healing. ${ }^{22}$ During the initial 15 minutes, a slower rate of formation appears to occur. After 15 minutes an increased rate of formation occurs tending towards a maximum around 30 minutes where the curve appears to plateau. Similar results have previously been reported, where after 25-30 minutes an effective steady state is reached between vacancy formation and healing. Both the overall behavior and trend appear to match that which was previously reported using the UV lamp, showing that the UV LED is an effective, useful and more practical alternative UV source. 


\section{CONCLUSION}

Rutile $\mathrm{TiO}_{2}$ thin films were combined with Au nanoparticles for use as a hybrid SERS substrates, utilizing the EM and chemical enhancement SERS mechanism. Additionally, photo-induced atomic oxygen vacancies were incorporated onto the surface of the films via UV irradiation. UV gas discharge lamps are a common choice for this purpose; however, these sources are often large, fragile, difficult to use experimentally and impractical to be incorporated into portable Raman systems safely. We have shown here how comparable effects and results can be obtained using UVC LED sources. Although there is a significant reduction in the source power, therefore a small reduction in SERS enhancement factor, this was not found to significantly affect the calculated dynamics of induced vacancies defects. A noticeable PIERS enhancement was still found and a clear change in measured signal in comparison to where no UV light was present. UV LEDs are more compact, versatile as well as being more cost effective. In contrast to discharge lamps and other UV sources, LEDs are far easier to incorporate into pre-existing Raman systems to allow real time enhancements to be monitored. Hence, we have shown that UV LED sources are a possible practical solution to allow fieldable PIERS measurements without compromising the measured Raman intensities significantly.

\section{REFERENCES}

[1] Tolaieb, B., Constantino, C. J. L. and Aroca, R. F., "Surface-enhanced resonance Raman scattering as an analytical tool for single molecule detection," Analyst 129(4), 337 (2004).

[2] Witlicki, E. H., Bähring, S., Johnsen, C., Solano, M. V., Nielsen, K. A., Silverstein, D. W., Marlatt, C. W., Jensen, L., Jeppesen, J. O. and Flood, A. H., "Enhanced detection of explosives by turn-on resonance Raman upon host-guest complexation in solution and the solid state," Chem. Commun. 53(10), 10918-10921 (2017).

[3] Yan, F. and Vo-Dinh, T., "Surface-enhanced Raman scattering detection of chemical and biological agents using a portable Raman integrated tunable sensor," Sensors Actuators, B Chem. 121(1), 61-66 (2007).

[4] Ben Jaber, S. S., Peveler, W. J., Quesada Cabrera, R., Sol, C. W. O., Papakonstantinou, I. and Parkin, I. P., "Sensitive and specific detection of explosives in solution and vapour by Surface-Enhanced Raman Spectroscopy on silver nanocubes," Nanoscale 9, 16459-16466 (2017).

[5] Le Ru, E. C., Blackie, E., Meyer, M., Etchegoin, P. G. and Etchegoint, P. G., "Surface Enhanced Raman Scattering Enhancement Factors: A Comprehensive Study," J. Phys. Chem. C 111, 13794 (2007).

[6] Schlücker, S., "Surface-Enhanced Raman Spectroscopy: Concepts and Chemical Applications," Angew. Chemie Int. Ed. 53(19), 4756-4795 (2014).

[7] Sharma, B., Frontiera, R. R., Henry, A.-I., Ringe, E. and Van Duyne, R. P., "SERS: Materials, applications, and the future," Mater. Today 15(1-2), 16-25 (2012).

[8] Le Ru, E. C. and Etchegoin, P. G. (Pablo G., [Principles of surface-enhanced Raman spectroscopy : and related plasmonic effects], Elsevier (2009).

[9] Otto, A., Mrozek, I., Grabhorn, H. and Akemann, W., "Surface-Enhanced Raman Scattering," J. Phys. Condens. Matter 4, 1143 (1992).

[10] Moskovits, M., "Surface-enhanced spectroscopy," Rev. Mod. Phys. 57(3), 783-826 (1985).

[11] Guerrini, L. and Graham, D., "Molecularly-mediated assemblies of plasmonic nanoparticles for SurfaceEnhanced Raman Spectroscopy applications," Chem. Soc. Rev. 41, 7085-7107 (2012).

[12] Ji, W., Zhao, B. and Ozaki, Y., "Semiconductor materials in analytical applications of surface-enhanced Raman scattering," J. Raman Spectrosc. 47(1), 51-58 (2016).

[13] Alessandri, I. and Lombardi, J. R., "Enhanced Raman Scattering with Dielectrics," Chem. Rev. 116(24), 1492114981 (2016).

[14] Lombardi, J. R. and Birke, R. L., "Theory of surface-enhanced raman scattering in semiconductors," J. Phys. Chem. C 118(20), 11120-11130 (2014).

[15] Wang, X., Shi, W., She, G. and Mu, L., "Surface-Enhanced Raman Scattering (SERS) on transition metal and semiconductor nanostructures," Phys. Chem. Chem. Phys. 14, 5891 (2012).

[16] Tan, X., Melkersson, J., Wu, S., Wang, L. and Zhang, J., "Noble-Metal-Free Materials for Surface-Enhanced Raman Spectroscopy Detection," ChemPhysChem 17, 2630-2639 (2016).

[17] Zheng, Z., Cong, S., Gong, W., Xuan, J., Li, G., Lu, W., Geng, F. and Zhao, Z., "Semiconductor SERS enhancement enabled by oxygen incorporation," Nat. Commun. 8, 1993 (2017).

[18] Cong, S., Yuan, Y., Chen, Z., Hou, J., Yang, M., Su, Y., Zhang, Y., Li, L., Li, Q., Geng, F. and Zhao, Z., "Noble metal-comparable SERS enhancement from semiconducting metal oxides by making oxygen vacancies," Nat. Commun. 6 (2015). 
[19] Han, X. X., Ji, W., Zhao, B. and Ozaki, Y., "Semiconductor-enhanced Raman scattering: Active nanomaterials and applications," Nanoscale 9, 4847 (2017).

[20] Ben-Jaber, S., Peveler, W. J., Quesada-Cabrera, R., Cortés, E., Sotelo-Vazquez, C., Abdul-Karim, N., Maier, S. A. and Parkin, I. P., "Photo-induced enhanced Raman spectroscopy for universal ultra-trace detection of explosives, pollutants and biomolecules," Nat. Commun. 7, 12189 (2016).

[21] Glass, D., Cortés, E., Ben-Jaber, S., Brick, T., Quesada-Cabrera, R., Peveler, W. J., Zhu, Y., Blackman, C. S., Howle, C. R., Parkin, I. P. and Maier, S. A., "Photo-induced enhanced Raman spectroscopy (PIERS): sensing atomic-defects, explosives and biomolecules ", Proc. SPIE 11010, Chemical, Biological, Radiological, Nuclear, and Explosives (CBRNE) Sensing XX, 110100D (17 May 2019);

[22] Glass, D., Cortés, E., Ben-Jaber, S., Brick, T., Peveler, W. J., Blackman, C. S., Howle, C. R., Quesada-Cabrera, R., Parkin, I. P. and Maier, S. A., "Dynamics of Photo-Induced Surface Oxygen Vacancies in Metal-Oxide Semiconductors Studied Under Ambient Conditions,” Adv. Sci., 1901841 (2019).

[23] D. W. Mayo, F. A. Miller, R. W. H., [Course Notes on the Interpretation of Infrared and Raman Spectra], John Wiley \& Sons, Inc., Hoboken, NJ, USA (2003). 